\title{
Cathepsin G-Induced Insulin-Like Growth Factor (IGF) Elevation in MCF-7 Medium Is Caused by Proteolysis of IGF Binding Protein (IGFBP)-2 but Not of IGF-1
}

\author{
Riyo Morimoto-Kamata,* Daiki Tsuji, and Satoru Yui \\ Laboratory of Host Defense, Faculty of Pharma-Science, Teikyo University; 2-11-1 Kaga, Itabashi-ku, \\ Tokyo 173-8605, Japan. \\ Received May 1, 2020; accepted August 2, 2020
}

Cathepsin G (CG), a neutrophil serine protease, induces cell migration and multicellular aggregation of human breast cancer MCF-7 cells. It has been suggested that tumor cell aggregates are associated with tumor embolism, thus CG-induced cell aggregation may promote tumor metastasis. We have revealed that cell aggregation is caused by elevated free insulin-like growth factor (IGF)-1 in the medium, followed by activation of IGF-1 receptor (IGF-1R). However, the molecular mechanism underlying IGF-1 elevation induced by CG remains unclear. Here, we aimed to elucidate the mechanism by examining the degradative effects of CG on IGF-1, and the IGF binding proteins (IGFBPs), which interfere with the binding of IGF-1 to its receptor. CG specifically evoked MCF-7 cell aggregation at less than $1 \mathrm{nM}$ in a dose-dependent manner, however, neutrophil elastase (NE), chymotrypsin, and trypsin did not. Free IGF-1 concentration was continuously elevated in the medium of cells treated with CG, whereas treatments with other serine proteases resulted in only a transient or slight increase. IGFBP-2, the predominant IGFBP in MCF-7 cells, was gradually digested by CG. CG did not cleave IGF-1 for at least $48 \mathrm{~h}$, whereas other proteases completely digested it. Moreover, CG induced continuous phosphorylation of IGF-1R and Akt, whereas NE-induced phosphorylation was transient, possibly due to insulin receptor substrate (IRS)-1 digestion. These results indicated that CG-specific IGF-1 elevation in the medium is caused by digestion of IGFBP-2, not IGF-1. Hence, this study clarifies the molecular mechanism of CG-specific cell aggregation.

Key words cathepsin G; breast cancer; insulin-like growth factor-1; insulin-like growth factor binding protein; serine protease

\section{INTRODUCTION}

In tumor tissues, infiltration of various non-transformed cells, including immune cells, fibroblasts, and vascular endothelial cells, occurs; they then interact with each other to compose the tumor microenvironment. ${ }^{1,2)}$ The infiltrating cells affect tumor proliferation and metastasis. Furthermore, intercellular communication in tumor tissues, mediated by direct contact and secretion of various factors, including cytokines, chemokines, growth factors, and inflammatory enzymes, is associated with tumor progression. Therefore, it is speculated that the infiltrating non-transformed cells can be potential targets for tumor suppression.

Neutrophils, which play a central role in innate immunity, are the most abundant type of granulocytes in most mammals and are frequently observed in various tumor tissues. Infiltrating neutrophils in tumor tissues, designated tumor-associated neutrophils (TANs), exert an anti-tumor effect, and therefore contribute to the elimination of tumor cells. ${ }^{3-5)}$ Recently, it has been revealed that TANs not only have anti-tumor activity but also pro-tumor activity. ${ }^{4,5)}$ N1-type TANs express an antitumor phenotype and suppress tumor progression by killing tumor cells through secreted cytokines, chemokines, and reactive oxygen species, whereas N2-type TANs promote tumor progression by tumor cell recruitment through chemokine signaling, the release of pro-growth and pro-invasion factors, and angiogenesis enhancement. Moreover, matrix metalloproteinase-9 (MMP-9), secreted by N2-type TANs, digests the extracellular matrix (ECM), thereby promoting the dissemination and metastasis of tumor cells into the surrounding tissues. Therefore, it is necessary to further elucidate the mechanism of tumor progression by N2-type TANs.

Neutrophil proteases stored in azurophil granules, such as cathepsin G (CG) and neutrophil elastase (NE), play an important role in eliminating invading microorganisms in the innate immune system. However, how neutrophil proteases contribute to tumor cell behavior remains unclear. We have previously revealed that $C G$ facilitates cell migration and the formation of E-cadherin-dependent multicellular aggregates of human breast cancer MCF-7 cells, followed by detachment from the culture substrate in an enzymatic activitydependent manner. ${ }^{6-8)}$ Since multicellular tumor aggregates cause tumor embolism and metastasis, it is suggested that CG may facilitate metastasis. As for its molecular mechanism, we previously demonstrated that CG-induced cell migration and aggregation are dependent on the activation of insulin-like growth factor (IGF)-1 receptor (IGF-1R). ${ }^{9}$ Specifically, silencing of IGF-1R gene expression using small interfering RNA and treatment with an anti-IGF-1R antibody, which targets the extracellular domain, or anti-IGF-1 antibody, eliminated CGinduced cell aggregation. Moreover, treatment of MCF-7 cells with CG elevated the levels of free IGF-1, which can then bind and activate IGF-1R in the conditioned medium. However, how CG elevates the free IGF-1 concentration in MCF-7 cell medium remains unresolved.

IGF-1 is a peptide hormone secreted from the liver and stim- 
ulated by growth hormones. It plays a critical role in mitogenesis, cell survival, and development. Following IGF-1 binding, IGF-1R undergoes autophosphorylation of its tyrosine kinase intracellular residues, resulting in activation of the adaptor protein insulin receptor substrate (IRS)-1, and downstream signaling molecules, including Akt, which is a serine/threoninespecific protein kinase. IGF-1 signaling is strongly correlated with breast cancer progression. ${ }^{10-13)}$ In fact, numerous clinical and epidemiological reports indicate a relationship between IGF-1 signaling and breast cancer. Indeed, breast cancer aberrantly expresses a component of the IGF-1 system ${ }^{11,12)}$ with IGF-1R immunoreactivity reported in $87 \%$ of human breast cancer tissues. ${ }^{14)}$ Both in vitro and in vivo models have indicated the role of the IGF-1 system in breast cancer via diverse endocrine, paracrine, and autocrine signaling pathways. ${ }^{12)}$

However, only a small amount of free IGF-1 is present under physiological conditions as a large proportion of it becomes bound to specific binding proteins, called IGF binding proteins (IGFBPs), and therefore most IGF-1 is prevented from binding to the receptor. IGFBPs, therefore, play important roles in IGF-1 signaling. ${ }^{11,15)}$ IGFBPs are specific carrier proteins comprised of six members, IGFBP-1 to -6 , all of which are organized into three domains that are conserved IGF binding domains in the $\mathrm{N}$ - and $\mathrm{C}$-termini and the linker domain that exists between the two binding domains. Additionally, IGFBPs have a higher affinity for IGF-1 than that between IGF-1R and IGF-1. ${ }^{13)}$ Therefore, in blood, 98\% of IGF-1 is bound to IGFBPs and since the IGF-1 and IGFBP complex cannot bind to the receptor, IGFBPs serve as negative regulators of IGF-1 signaling. Moreover, the level of free IGF-1 is regulated by IGFBP proteolysis as cleaved IGFBP reduces its affinity for IGF-1. ${ }^{16-18)}$ IGFBPs are digested by various proteases including kallikreins, MMPs, and cathepsins. ${ }^{19,20)}$ Thus, it is suggested that proteases affecting IGFBP are direct regulators in IGF-1 signaling.

Here, we hypothesize that the elevation of free IGF-1 in conditioned medium of MCF-7 cells after CG treatment is caused by IGFBP proteolysis. Interestingly, Gibson and Cohen ${ }^{21)}$ reported that $\mathrm{CG}$ and NE are capable of cleaving IGFBPs in vitro. In addition, their work demonstrates that $\mathrm{NE}$ digests recombinant IGF-1 within $20 \mathrm{~min}$, meanwhile CG does not have the same effect after $1 \mathrm{~h}$. We hypothesize that CG-specific IGF-1 upregulation is derived from degradation of IGFBP, not IGF-1; whereas NE digests both. To test this hypothesis, we compared $\mathrm{CG}$ with $\mathrm{NE}$ and pancreatic proteases chymotrypsin, and trypsin in terms of MCF-7 cell aggregation activity and proteolytic activity against IGFBP and IGF-1. IGFBP-2, which is a unique IGFBP member expressed in MCF-7 cells, is gradually fragmented by CG. Meanwhile, other serine proteases rapidly digested IGFBP-2. As for the ability to digest recombinant IGF-1, other serine proteases quickly digested IGF-1 in vitro, whereas $\mathrm{CG}$ did not. We conclude that $\mathrm{CG}$ specifically increases free IGF-1 in the medium of MCF-7 cells via IGF-1 release through IGFBP-2 proteolysis, however, not through direct IGF-1 proteolysis. In this study, we revealed the molecular mechanism for the CG-specific increase in free IGF-1.

\section{MATERIALS AND METHODS}

Reagents $\mathrm{CG}$ and NE purified from human neutrophils were obtained from BioCentrum (Krakow, Poland) and Merck
Millipore (Burlington, MA, U.S.A.), respectively. Bovine pancreas chymotrypsin, trypsin and human $\alpha 1$-antitrypsin were purchased from Sigma-Aldrich (St. Louis, MO, U.S.A.). Anti-IGFBP-2 (Cat. No. 3922), anti-IGF-1R $\beta$-subunit (Cat. No. 9750), anti-IRS-1 (Cat. No. 2382), anti-phospho-Akt antibodies (Cat. No. 4060), and anti-Akt antibodies (Cat. No. 9272) were purchased from Cell Signaling Technology (Danvers, MA, U.S.A.), whereas anti-IGF-1 antibodies (Cat. No. ab9572) were obtained from Abcam (Cambridge, U.K.). Anti-phosphotyrosine (Cat. No, 05-321) antibodies were purchased from Merck Millipore.

Cells Human breast cancer MCF-7 cells were provided by Dr. Hiroshi Kosano (Teikyo University, Japan) and were maintained in RPMI 1640 medium, supplemented with 10\% heatinactivated fetal bovine serum (FBS; MP Biomedicals, Solon, OH, U.S.A.) and $80 \mu \mathrm{g} / \mathrm{mL}$ kanamycin (Wako Pure Chemical Corporation, Osaka, Japan), as described previously. ${ }^{9)}$

Serine Protease Treatment To induce cell aggregation, MCF-7 cells were treated with serine proteases. Briefly, MCF-7 cells $\left(1 \times 10^{4}\right.$ cells/well $)$ were seeded in 96 -well plates in RPMI 1640 medium containing 5\% FBS and then washed with serum-free RPMI 1640 medium. After cultivation for $24 \mathrm{~h}$, the cells were pre-cultured for $1 \mathrm{~h}$ in RPMI 1640 containing $1 \%$ bovine serum albumin (BSA). Subsequently, the cells were incubated with purified serine proteases for $24 \mathrm{~h}$. Cell morphology was observed by a microscopy Olympus CKX 41 (Tokyo, Japan).

Quantification of IGF-1 and IGFBP-2, -4, and -5 The concentrations of IGF-1 and IGFBPs in the conditioned media were determined using an enzyme-linked immunosorbent assay (ELISA) kit according to the manufacturer's instructions. The conditioned medium was harvested after a 24-h cultivation in RPMI 1640 containing 1\% BSA. The free IGF-1 was quantified under nondenaturing conditions. ELISA kits for IGF-1, IGFBP-2, and -4 were purchased from R\&D Systems, whereas that for IGFBP-5 was obtained from Thermo Fisher Scientific (Waltham, MA, U.S.A.). To prevent the proteolysis of IGF-1 and IGFBPs by serine proteases, a protease-inhibitor cocktail (cOmplete; Roche Diagnostics, Mannheim, Germany) was added to the harvested conditioned medium. Cell debris was removed by centrifugation at $500 \times \boldsymbol{g}$ for $5 \mathrm{~min}$.

Western Blotting Sodium dodecyl sulfate (SDS)polyacrylamide gel electrophoresis and subsequent Western blotting analyses were performed as previously described.9 After removing cell debris, the harvested conditioned medium obtained from MCF-7 cells was supplemented with the protease-inhibitor cocktail. Samples were then combined with $5 \times$ sample buffer $(300 \mathrm{mM}$ Tris- $\mathrm{HCl}, \mathrm{pH} 6.8)$ containing $10 \%$ 2-mercaptoethanol, $10 \%$ SDS, 50\% glycerol, and $0.05 \%$ Coomassie brilliant blue at a $1: 4$ ratio and boiled for $5 \mathrm{~min}$. The resulting samples were subsequently separated by SDS-PAGE (20 $\mu \mathrm{L} /$ lane) using pre-cast $5-20 \%$ Tris-glycine gradient gels (Atto Corporation, Tokyo, Japan). For IGF-1 detection, 16.5\% Tris-Tricine gel was used. The separated samples in gels were transferred to polyvinylidene difluoride membranes (Wako Pure Chemical Corporation). Membranes were blocked by incubation with Tris-buffered saline (TBS) containing $0.1 \%$ Tween 20 (TBS-T) and 5\% skim milk (Megmilk Snow Bland, Tokyo, Japan) or BSA (Sigma-Aldrich) for $1 \mathrm{~h}$, probed with the appropriate primary antibodies, diluted with Can Get Signal Immunoreaction Enhancer Solution (Toyobo, Osaka, Japan) 


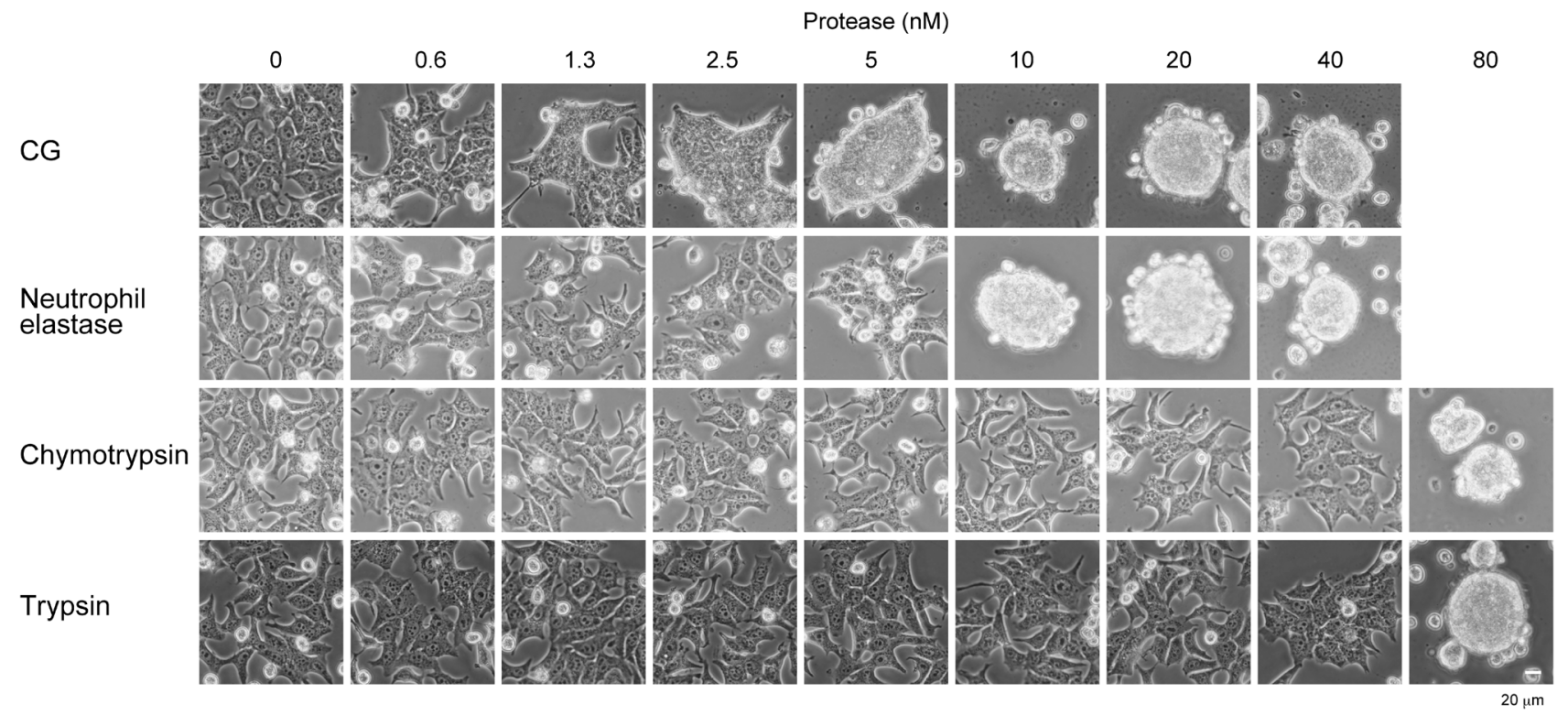

Fig. 1. Cathepsin G Specifically Enhances MCF-7 Cell Migration at Lower Concentrations than Neutrophil Elastase, Chymotrypsin, and Trypsin

MCF-7 cells were inoculated in RPMI 1640 medium containing 5\% FBS. The cells were treated with cathepsin G (CG), neutrophil elastase (NE), chymotrypsin, or trypsin for $24 \mathrm{~h}$.

at $4^{\circ} \mathrm{C}$, washed extensively with TBS-T, and incubated with horseradish peroxidase (HRP)-conjugated secondary antibodies (Cytiva, Tokyo, Japan; 1:5000 dilution). Finally, the membranes were washed, developed by incubation with enhanced chemiluminescence (ECL) detection reagents (Cytiva), and exposed to Hyperfilm ECL (Cytiva).

Recombinant IGF-1 Proteolysis Recombinant human IGF-1 ( $2 \mu \mathrm{M}$, Wako Pure Chemical Corporation) was incubated with tserine proteases (CG and NE, $40 \mathrm{nM}$; chymotrypsin and trypsin, $80 \mathrm{nM}$ ) for $48 \mathrm{~h}$. IGF-1 fragmentation was detected by Western blotting using an anti-IGF-1 antibody (Abcam).

Immunoprecipitation Immunoprecipitation was performed as described previously. ${ }^{9)}$ Briefly, whole cell lysates were prepared by incubating cells with Pierce IP Lysis Buffer (Thermo Fisher Scientific) for $15 \mathrm{~min}$ on ice. The lysates $(500 \mu \mathrm{g})$ were then incubated with the anti-IGF-1R $\beta$-subunit antibody $(5 \mu \mathrm{L})$ overnight at $4{ }^{\circ} \mathrm{C}$, after which the antibody was bound to Protein A-Sepharose (Cytiva) at $4{ }^{\circ} \mathrm{C}$ for $1 \mathrm{~h}$. After formation of the antibody-bead complex, the beads were washed thrice with lysis buffer, and the captured protein complex was eluted in $3 \times$ sample buffer $(180 \mathrm{mM}$ Tris $-\mathrm{HCl}[\mathrm{pH}$ 6.8] containing $6 \% 2$-mercaptoethanol, $6 \%$ SDS, $30 \%$ glycerol and $0.03 \%$ Coomassie brilliant blue). Eluates were analyzed by immunoblot using the anti-phosphotyrosine antibody.

\section{RESULTS}

CG Induced MCF-7 Cell Aggregation in a DoseDependent Manner, While NE and Pancreatic Serine Proteases Required Higher Concentrations We have previously revealed that CG facilitated MCF-7 cell migration and multicellular aggregate formation. ${ }^{6-9)} \mathrm{NE}$, pancreatic chymotrypsin, and trypsin also induced the formation of MCF-7 spheroids, however, did not facilitate their migration. ${ }^{22)}$ The spheroid formation-inducing activity of NE, chymotrypsin, and trypsin appears to be primarily dependent on degradation of the adhesion molecules on culture substrate, such as fibronec- tin. However, the digestion of fibronectin by chymotrypsin and trypsin required a higher concentration compared to that for CG and NE. ${ }^{22)}$ To compare the effect of dose on induction of cell aggregation, MCF-7 cells were treated with CG, NE, chymotrypsin or trypsin (Fig. 1). CG induced cell aggregation at a dose of less than $1 \mathrm{nM}$, whereas NE induced cell aggregation at more than $10 \mathrm{nM}$. Chymotrypsin and trypsin formed spheroids at $80 \mathrm{nM}$. These results indicate that CG specifically facilitates MCF-7 cell aggregation in a dose-dependent manner.

CG Treatment Evokes Elevation of Free IGF-1 in the Conditioned Medium of MCF-7 Cells We have previously demonstrated that IGF-1 signaling is required for CG-induced cell aggregation, and that $\mathrm{CG}$ treatment of MCF-7 cells evokes elevated IGF-1 levels in the conditioned medium. ${ }^{9)} \mathrm{We}$, therefore next sought to examine whether other proteases similarly increase IGF-1 levels. To this end, we quantified the concentration of free IGF-1 in conditioned medium treated with other serine proteases. Since free IGF-1 could activate IGF-1R, we determined free IGF-1 concentration in the conditioned medium under nondenaturing conditions. When treated with CG, free IGF-1 levels were continuously elevated in the conditioned medium for $4 \mathrm{~h}$, with a large proportion maintained at $24 \mathrm{~h}$ (Fig. 2). Alternatively, upon treatment with NE, IGF-1 levels increased within $1 \mathrm{~h}$, however, decreased thereafter. Meanwhile, IGF-1 concentration upon treatment with chymotrypsin increased after $0.25 \mathrm{~h}$ yet rapidly decreased to the level of vehicle-treated cells thereafter. With trypsin treatment, IGF-1 slightly increased after $1 \mathrm{~h}$ but then decreased to basal concentration. Of note, we confirmed that CG treatment did not alter IGF-1 contained in the lysate of MCF-7 cells using Western blotting (Supplementary Fig. 1). Thus, CG did not affect IGF-1 expression. Further, following inhibition of CG enzymatic activity by addition of $\alpha 1$-antitrypsin at $1 \mathrm{~h}$, the cell aggregation induced by CG was sustained up to $4 \mathrm{~h}$, and was observed at a lower level at $24 \mathrm{~h}$ (Supplementary Fig. 2). These data suggest that CG-induced cell migration is associated with IGF-1 release via enzymatic activity. Overall, only MCF-7 


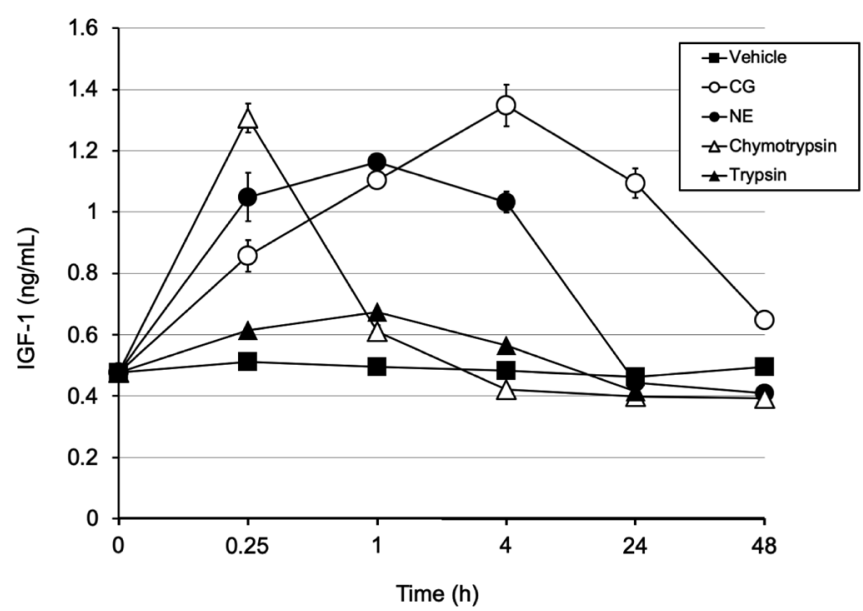

Fig. 2. Cathepsin G Evokes Continuous Elevation of Free InsulinLike Growth Factor 1 in the Conditioned Medium, Whereas Neutrophil Elastase, Chymotrypsin, and Trypsin Did Not

The concentration of free insulin-like growth factor (IGF)-1 in the conditioned medium treated with a serine protease was determined by enzyme-linked immunosorbent assay (ELISA). The cells were treated with cathepsin G (CG; 40nM), neutrophil elastase $(\mathrm{NE} ; 40 \mathrm{nM})$, chymotrypsin $(80 \mathrm{nM})$, or trypsin $(80 \mathrm{nM})$ for $24 \mathrm{~h}$. The results are presented as means \pm standard deviation $(n=3)$.

cells treated with CG had continuously increased free IGF-1 concentration in the medium. Although other proteases either briefly increased free IGF-1 concentration or had little effect.

Among IGFBP Isoforms, IGFBP-2 Is Dominant in the Conditioned Medium of MCF-7 Cells It has been reported that MCF-7 cells express mRNAs for IGFBP-2, -4 , and $-5 .^{23)}$ We, therefore, determined the concentration of IGFBP-2, -4, and -5 in the conditioned medium under untreated conditions, as well as with IGFBP-3 as this is the dominant member found in the blood. IGFBP-2 was detected at an average concentration of $12.0 \pm 0.48 \mathrm{ng} / \mathrm{mL}$ in the conditioned medium (Table 1), whereas IGFBP-4, -5 , and -3 were under the detection limit for ELISA. Furthermore, IGFBP-2 in the medium was detected using Western blotting, whereas IGFBP-4 and -5 were not (data not shown). Therefore, we concluded that IGFBP-2 is the primary IGFBP member found within the conditioned medium of MCF-7 cells.

IGFBP-2 Is Slowly Digested by CG and NE, However, Is Rapidly Digested by Chymotrypsin and Trypsin Subsequently, we examined whether the serine proteases can digest IGFBP-2. Vehicle-treated cell medium contained intact IGFBP-2, which was $35 \mathrm{kDa}$ in size (Fig. 3). IGFBP-2 contained in the medium of CG-treated cells was fragmented to 13 and $10 \mathrm{kDa}$ at $4 \mathrm{~h}$ after treatment, and these fragments were not detected after $24 \mathrm{~h}$. Similarly, NE treatment also digested IGFBP-2 into $13-\mathrm{kDa}$ fragments at $4 \mathrm{~h}$ after treatment. In contrast, pancreatic chymotrypsin and trypsin rapidly degraded IGFBP-2, resulting in undetectable levels of intact IGFBP-2 within $0.25 \mathrm{~h}$. Meanwhile, the expression levels of IGFBP-2 protein in CG-treated cells were the same as that of vehicletreated cells at $1 \mathrm{~h}$ and $4 \mathrm{~h}$, which then slightly decreased at 24h (Supplementary Fig. 3). These results indicated that CG and NE slowly digest IGFBP-2 compared to chymotrypsin and trypsin.

Free IGF-1 Is Not Cleaved by CG, However, Is Cleaved by Other Serine Proteases IGFBP-2 proteolysis releases free IGF-1. However, IGF-1 might also be degraded by serine proteases considering that IGF-1 is a peptide hormone and
Table 1. Concentration of Insulin-Like Growth Factor Binding Protein (IGFBP)-2, -3, -4, and -5 in Conditioned MCF-7 Cell Medium during Basal Condition

\begin{tabular}{cc}
\hline \hline & Concentration $(\mathrm{ng} / \mathrm{mL})$ \\
\hline IGFBP-2 & $12.0 \pm 0.48$ \\
IGFBP-3 & Under detection limit $(<0.781)$ \\
IGFBP-4 & Under detection limit $(<3.59)$ \\
IGFBP-5 & Under detection limit $(<3.29)$ \\
\hline
\end{tabular}

Concentration of IGFBPs in the conditioned media were determined using an enzyme-linked immunosorbent assay (ELISA) kit according to the manufacturer's instructions. The conditioned medium was harvested after 24-h cultivation in RPMI 1640 containing $1 \%$ BSA.

may be a substrate of these proteases. We, therefore, investigated whether these serine proteases could digest recombinant human IGF-1 in vitro as we failed to detect free IGF-1 and IGFBP-bound IGF-1 in the medium separately using SDSPAGE, followed by Western blotting. CG had little effect on IGF-1 cleavage at least for $48 \mathrm{~h}$ (Fig. 4), whereas NE digested almost all IGF-1 within $4 \mathrm{~h}$. Treatments with chymotrypsin or trypsin resulted in undetectable intact IGF-1 after $0.25 \mathrm{~h}$. These data suggest that CG released IGF-1 by IGFBP-2 proteolysis, whereas NE, chymotrypsin, and trypsin digested both IGFBP-2 and IGF-1.

NE-Induced IGF-1 Signaling Is Transient Due to IRS-1 Proteolysis Finally, we examined how long IGF-1 signaling is sustained by CG and NE treatment since NE induced similar maximum levels of IGF-1 release as was observed by CG, in the early stage (Fig. 2). Results show that CG and NE both induced IGF-1R phosphorylation for $4 \mathrm{~h}$ after treatment (Fig. 5A). Moreover, considering that NE becomes incorporated into tumor endosomes via clathrin pit-dependent endocytosis, where it has been reported to decompose IRS-1 in lung adenocarcinomas, ${ }^{24,25)}$ we next investigated intracellular IRS-1 protein levels following $\mathrm{CG}$ and $\mathrm{NE}$ treatment. $\mathrm{CG}$ did not alter IRS-1 protein levels, however, was found to stimulate Akt phosphorylation for $4 \mathrm{~h}$ (Figs. 5B, C). Alternatively, NE decreased IRS-1 protein, yet only induced phosphorylation of Akt for $1 \mathrm{~h}$ after treatment (Fig. 5C). Meanwhile, chymotrypsin did not induce IGF-1R phosphorylation (Fig. 5A). Curiously, trypsin induced IGF-1R phosphorylation for $4 \mathrm{~h}$, however, intact IGF-1 was not detected $0.25 \mathrm{~h}$ after treatment (Fig. 4). Moreover, after $4 \mathrm{~h}$ trypsin did not cause IRS-1 digestion or Akt phosphorylation (Fig. 5). Collectively, these results suggest that CG continuously activates IGF-1 signaling, while NE rapidly inhibits signaling via proteolysis of IGF-1 and IRS-1.

In summary, CG-induced continuous elevation of free IGF-1 likely via slow IGFBP-2 degradation and non-degradation of IGF-1. Alternatively, NE, chymotrypsin and trypsin rapidly decomposed both IGFBP-2 and IGF-1.

\section{DISCUSSION}

In our previous study, we demonstrated that CG-induced MCF-7 cell migration and aggregation are responsible for the activation of IGF-1 signaling involved in increasing IGF-1 levels. ${ }^{9)}$ However, the molecular mechanism responsible for how CG evokes the elevated IGF-1 levels in conditioned media remained unclear. Here, we determined that this increase is caused by proteolysis of IGFBP-2, not of IGF-1. Furthermore, higher concentrations of other serine proteases, namely NE, 

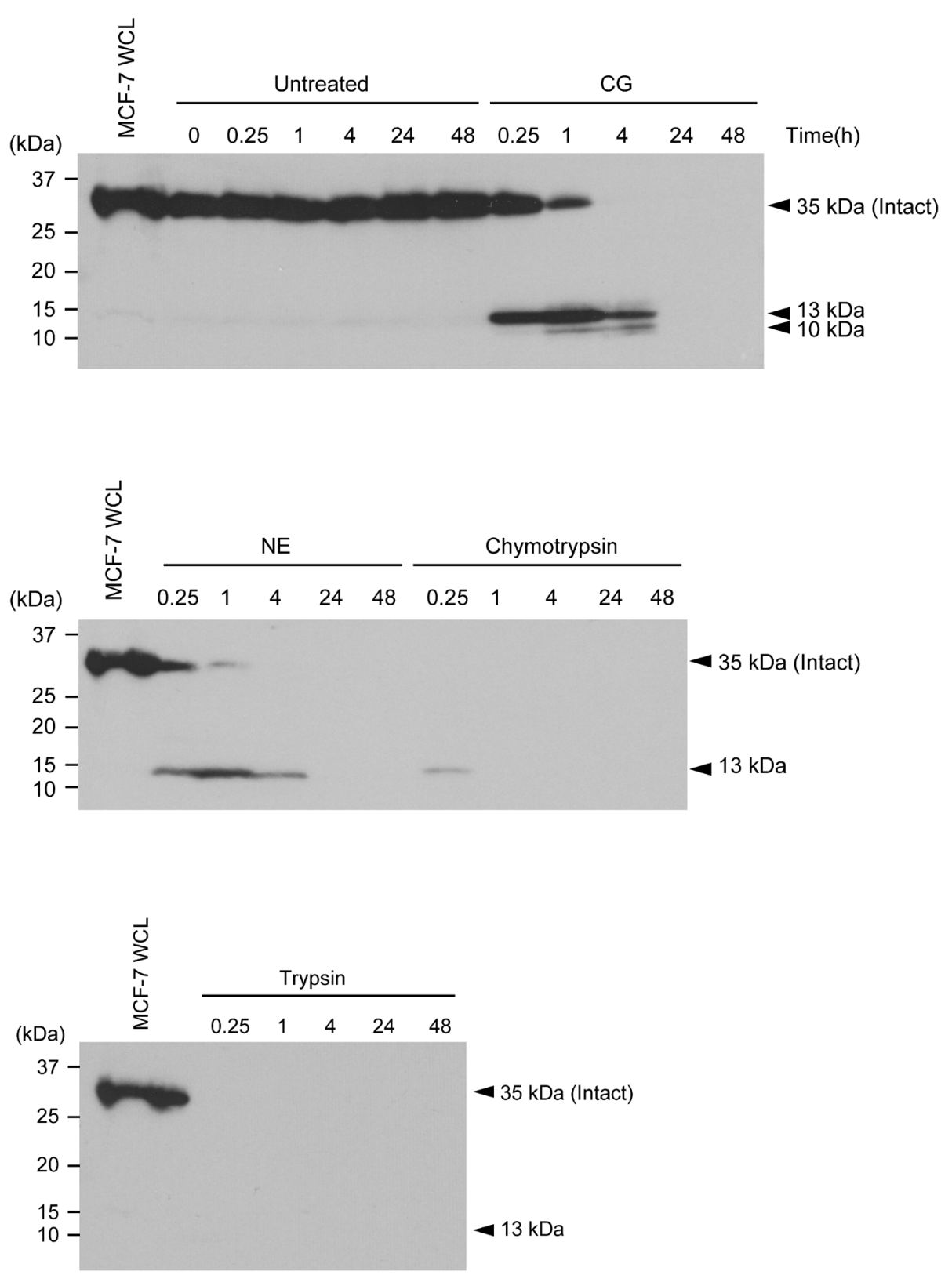

Fig. 3. Insulin-Like Growth Factor Binding Protein 2 Is Gradually Processed by Cathepsin G and Is Rapidly Digested by Other Serine Proteases

Insulin-like growth factor binding protein 2 (IGFBP-2) in the conditioned medium ( $20 \mu \mathrm{L} /$ lane) of MCF-7 cells treated with a serine protease [cathepsin G (CG) and neutrophil elastase (NE), $40 \mathrm{nM}$; chymotrypsin and trypsin, $80 \mathrm{nM}$ ] was detected by Western blot analysis using an anti-IGFBP-2 polyclonal antibody. Intact IGFBP-2 ( $35 \mathrm{kDa})$ and its fragments are indicated. Whole cell lysate (WCL, $20 \mu \mathrm{g}$ ) was used as the positive control.

chymotrypsin, and trypsin, were required to induce cell aggregation compared to that observed with CG. We also demonstrated that this requirement for higher concentrations of $\mathrm{NE}$, chymotrypsin and trypsin is caused by the breakdown of adhesion molecules in the culture substrates rather than as the result of cell-oriented mechanisms. ${ }^{22)}$ Moreover, these other serine proteases did not stimulate cell migration. ${ }^{9)}$ However, NE was found to transiently increase the level of free IGF-1 in the conditioned medium at nearly the same level as that observed with CG during the early phase, and evoked IGF-1R activation for $4 \mathrm{~h}$ (Figs. 2, 5A). Meanwhile, IGF-1R activation by $\mathrm{NE}$ is not believed to make a significant contribution to cell aggregation as NE also transiently induced Akt phosphorylation via IRS-1 proteolysis (Fig. 5). Hence, we concluded that NE-stimulated spheroid formation is primarily induced by digestion of adhesion molecules and ECM.
We previously showed that the digestion of ECM components, such as fibronectin, by pancreatic chymotrypsin and trypsin is less effective than that by $\mathrm{CG}$ and NE. ${ }^{22)}$ Therefore, chymotrypsin and trypsin might require much higher concentrations to aggregate MCF-7 cells. This study suggests that chymotrypsin does not induce activation of IGF-1 signaling due to the rapid degradation of IGF-1. Moreover, trypsin is unable to induce IGF-1R activation via ligand binding; since despite the fact that trypsin phosphorylates IGF-1R, it also rapidly digests IGF-1. Insulin receptor family members, including IGF-1R, have unique conformations among receptor tyrosine kinases in forming constitutive dimers in the absence of ligand. ${ }^{26,27)}$ Dimerization of the insulin receptor in the absence of ligand cannot induce an activation signal; rather it contributes to maintaining the inactive state, and a ligand dependent conformational change is required for kinase activity 

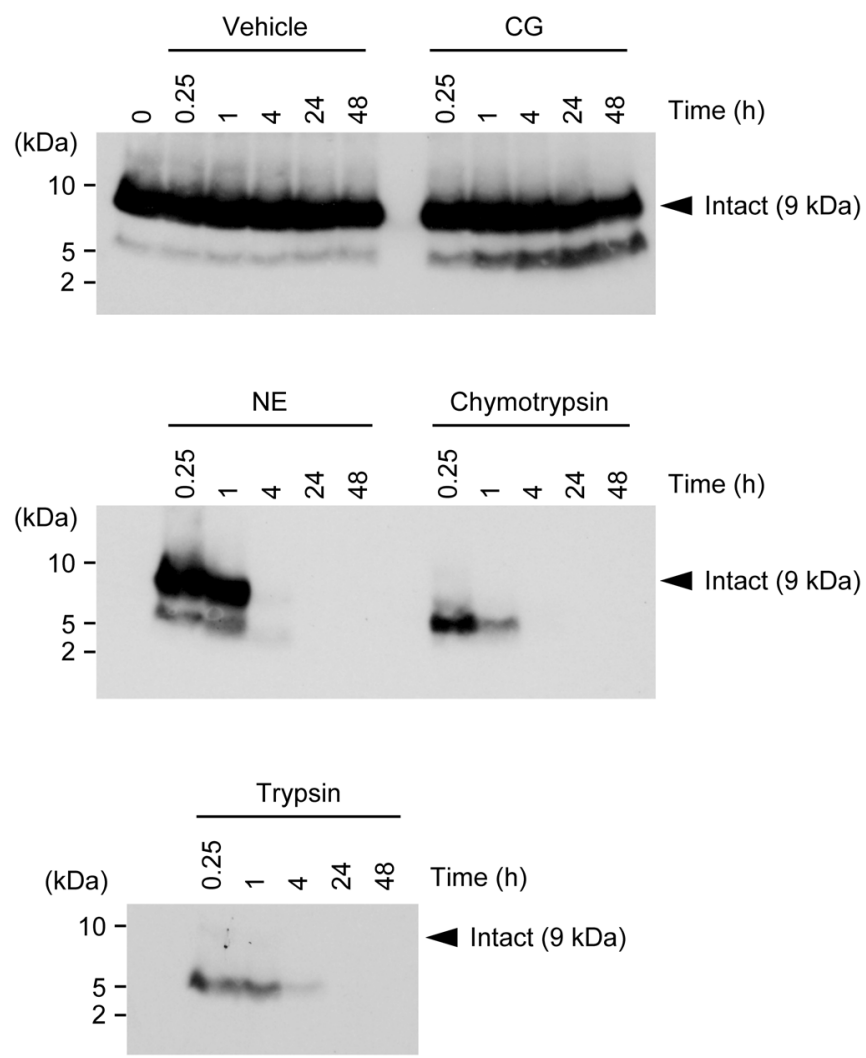

Fig. 4. Free Insulin-Like Growth Factor (IGF)-1 Is Not Digested by Cathepsin G in Vitro for after $48 \mathrm{~h}$ but Was Rapidly Digested by Neutrophil Elastase, Chymotrypsin, and Trypsin

Recombinant human IGF-1 $(2 \mu \mathrm{M})$ and a serine [cathepsin $\mathrm{G}(\mathrm{CG})$ and neutrophil elastase (NE), $40 \mathrm{nM}$; chymotrypsin and trypsin, $80 \mathrm{nM}$ ] were incubated for the indicated time period. IGF-1 was detected by Western blotting using anti-IGF-1 polyclonal antibody.

of the receptor intracellular domain. ${ }^{28)}$ Alternatively, tryptic removal of the insulin receptor extracellular domain results in constitutive tyrosine phosphorylation without activation of its kinase. Hence, trypsin induced phosphorylation of IGF-1R but not Akt.

Whether IGF-1 serve as substrate for these serine proteases and degradation efficiency of IGFBP-2 and IGF-1 by these proteases differ depending on the protease. We postulate that the slow decomposition of IGFBP-2 by CG compared to the other proteases, is due to substrate specificity. CG has broad specificity that favors large hydrophobic (phenylalanine (Phe), leucine (Leu), and methionine (Met)) and positive (lysine (Lys) and arginine (Arg)) P1 residues when synthetic peptides are the substrates. ${ }^{29-33)}$ In addition to $\mathrm{P} 1$ residue, the substrate specificity for proteins of CG may be affected by the conformation and/or the residues surrounding the P1 residue compared to the other proteases. Indeed, CG did not exhibit a remarkable degree of recombinant human IGF-1 cleavage, although recombinant IGF-1 used in our study contained 4, 6, and 2 residues of Phe, Leu, and Met, respectively. Further, the recombinant IGF-1 had positive residues, i.e., 3 and 6 of Lys and Arg, respectively.

Recent reports suggest that NE is a malignant factor in various types of cancer, including breast cancer. ${ }^{24,34,35)}$ Some reports have also indicated that NE is associated with tumor progression through the IGF-1-independent pathway. NE accelerates cancer cell proliferation in lung cancer cells via
IRS-1 degradation followed by increasing the interaction between phosphatidylinositol 3-kinase and platelet-derived growth factor receptor. ${ }^{24)} \mathrm{NE}$ cooperates with MMP-9 to promote tumor cell dissemination and metastasis via breakdown of the basement membrane and subsequent ECM and vascular endothelial growth factor (VEGF) release. ${ }^{34)}$ In fact, experimental and clinical studies have suggested that higher NE concentration in primary breast cancer correlates with poor prognosis. ${ }^{35)}$ In contrast, few reports have investigated the role of CG in tumors; therefore, it is unclear whether CG is associated with tumor progression. $\mathrm{CG}$ induces cell aggregation of limited cell types, including mammary tumor cell lines, such as MCF-7, BT474, and T47D cells, which are E-cadherin and IGF-1R positive. ${ }^{9)}$ However, CG did not induce cell aggregation, migration, nor detachment in the human HeLa and sarcoma HT1080 cervical cancer lines, irrespective of IGF$1 \mathrm{R}$ expression (unpublished data). The mechanism by which $\mathrm{CG}$ induces cell aggregation in specific cell lines is not yet understood. IGF-1 signaling is constituted by many members such as IGF-1, binding proteins, proteases degrading IGFBPs, and IGF-1 receptors. Variation in the expression patterns of the components of IGF-1 signaling among cell lines can lead to differences in $\mathrm{CG}$ response. For example, estrogen receptor (ER) status directly correlates with IGFBP2 expression in breast cancer, with ER-positive cancers, such as MCF-7, having higher levels of IGFBP2 than ER-negative cancers, such as MDA-MB-231. ${ }^{36-38)}$ Moreover, in MDA-MB-231 cells, CG did not induce cell aggregation. ${ }^{22}$ These differences may determine whether $\mathrm{CG}$ induces cell aggregation. We are, therefore, working to elucidate the mechanism of specific cell aggregation by $\mathrm{CG}$. Both CG and NE are stored in azurophil granules, and co-secreted from activated neutrophils. Although NE digests free IGF-1, we speculate that CG-induced cell aggregation occurs under pathophysiological conditions considering that cell lysates prepared from rat neutrophils, containing $\mathrm{CG}$ and NE, induced cell aggregating activity in a dosedependent manner in a similar manner observed with purified CG protein. ${ }^{6}$ Moreover, the enzymatic activity of CG in cell lysates prepared from rat neutrophils, and in the supernatant of formyl-methionyl-leucyl-phenylalanine plus cytochalasin B-activated rat neutrophils, was 10-fold higher than that of NE (3.1 and $0.37 \mathrm{mU} / 2.5 \times 10^{6}$ cells, 2.5 and $0.19 \mathrm{mU} / \mathrm{mL}$, respectively). ${ }^{6}$ We, therefore, believe that $\mathrm{CG}$ induces breast cancer cell aggregation via IGF-1 signaling in the pathophysiological condition.

IGF-1 is an important mitogen in the mammary gland. ${ }^{10,16,39-41)}$ Many epidemiological and prospective studies have attempted to confirm the positive correlation between plasma IGF-1 levels and breast cancer risk. ${ }^{12)}$ Additionally, most studies suggest that elevated systemic or tissue levels of IGFBP are found in breast cancer. Sufficient experimental evidence implicates IGFBPs in the pathophysiology of breast cancer, particularly IGFBP-2, -3 , and -5 . Using ELISA, we showed that only the IGFBP-2 protein is detectable in the conditioned MCF-7 medium before stimulation although transcriptional expression of IGFBP-2, -4 , and -5 in the cells was reported ${ }^{23)}($ Table 1$)$. However, there are many conflicting reports regarding the role of IGFBP-2 in tumor progression. ${ }^{42}$ Epidemiological studies have shown that tissue IGFBP-2 is upregulated at the protein level and is correlated with tumor progression in many cancers, including breast cancer, ${ }^{12,38,43)}$ 


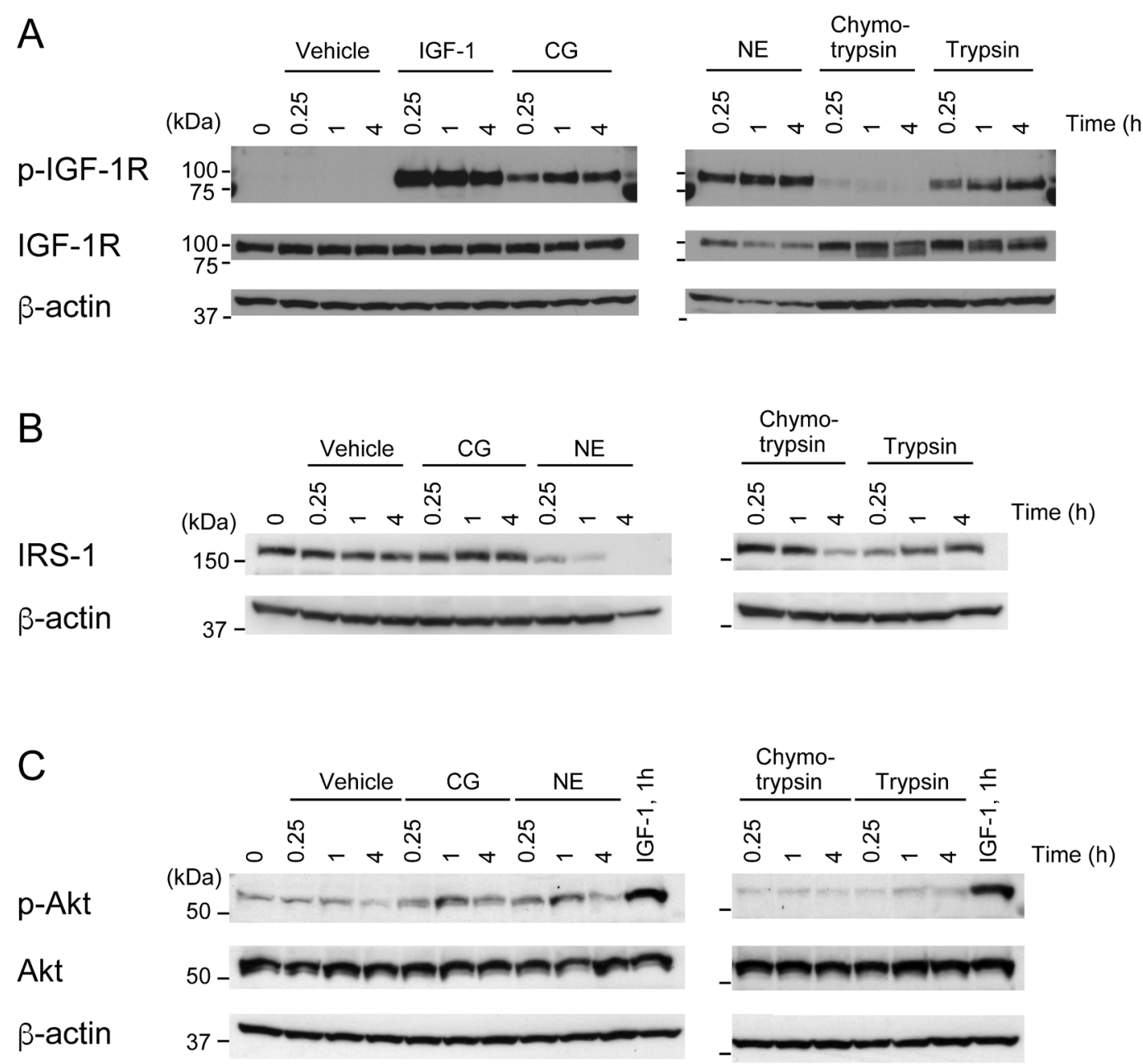

Fig. 5. Cathepsin G Induces Continuous Phosphorylation of Insulin-Like Growth Factor-1 Receptor and Akt, While Neutrophil Elastase Transiently Phosphorylated Akt Due to Insulin Receptor Substrate-1 Proteolysis

MCF-7 cells were treated with serine proteases [cathepsin G (CG) and neutrophil elastase (NE), 40 nM; chymotrypsin and trypsin, $80 \mathrm{nM}$ ]. (A) Tyrosine phosphorylated insulin-like growth factor-1 receptor (p-IGF-1R) in the MCF-7 cells treated with serine proteases was detected by immunoprecipitation using an anti-IGF-1R antibody and subsequent Western blotting with an anti-phosphotyrosine antibody. (B) Insulin receptor substrate-1 (IRS-1) protein in the MCF-7 cells treated with serine proteases was detected by Western blotting using an IRS-1 antibody. (C) Phosphorylated Akt contained in the MCF-7 cells treated with serine proteases was detected by Western blotting using an anti-phospho-Akt (p-Akt) antibody. The cell lysates were analyzed at $20 \mu \mathrm{g} / \mathrm{lane}$.

and is significantly expressed in breast cancer compared to benign lesions. ${ }^{44)}$ However, IGFBP-2 expression did not correlate with the stage of disease in ovarian cancer, with similar proportions of tumors observed to express low and high levels of IGFBP2 at all disease stages. ${ }^{45)}$ Nevertheless, IGFBP-2 is proposed to suppress tumor development through binding IGFs, thereby preventing binding to their receptor and subsequent IGF-driven tumorigenesis and tumor progression. ${ }^{10,40,41)}$ Despite the suppressive function of IGFBP-2, there are also studies that demonstrate its oncogenic functions, including promoting proliferation, driving VEGF expression, and suppressing apoptosis via an IGF-1-independent mechanism. ${ }^{46,47)}$ At minimum, IGFBP-2 cleavage by CG prevents IGF-1-dependent functioning. This study will help elucidate these contradictions, particularly regarding the role of IGFBP proteases in tumor progression.

In summary, the increased level of free IGF-1 evoked by $\mathrm{CG}$ is caused by release of IGF-1 from IGFBP-2 following CG digestion of IGFBP-2, rather than direct digestion of IGF-1. This study presents a novel molecular mechanism underlying the upregulation of tissue IGF-1 levels in breast cancer MCF-7 cells by a neutrophil protease. Further, we propose that TANs contribute to tumor metastasis and dissemination through an IGF-1-dependent mechanism. We believe that the results of this study will serve to inform the development of new drugs to prevent tumor metastasis mediated by invasive neutrophils.

Acknowledgments This work was supported by a KAKENHI Grant (Grant No. JP15K18417 to RMK) from Ministry of Education, Culture, Sports, Science and Technology (MEXT) of Japan.

Conflict of Interest The authors declare no conflict of interest.

Supplementary Materials The online version of this article contains supplementary materials.

\section{REFERENCES}

1) Yuan Y, Jiang YC, Sun CK, Chen QM. Role of the tumor microenvironment in tumor progression and the clinical applications. Oncol. Rep., 35, 2499-2515 (2016).

2) Balkwill FR, Capasso M, Hagemann T. The tumor microenviron- 
ment at a glance. J. Cell Sci., 125, 5591-5596 (2012).

3) Powell DR, Huttenlocher A. Neutrophils in the tumor microenvironment. Trends Immunol., 37, 41-52 (2016).

4) Kim J, Bae JS. Tumor-associated macrophages and neutrophils in tumor microenvironment. Mediators Inflamm., 2016, 6058147 (2016).

5) Shaul ME, Fridlender ZG. Cancer-related circulating and tumorassociated neutrophils-subtypes, sources and function. FEBS J., 285, 4316-4342 (2018).

6) Yui $\mathrm{S}$, Tomita $\mathrm{K}, \mathrm{Ku}$ do $\mathrm{T}$, Ando $\mathrm{S}$, Yamazaki M. Induction of multicellular 3-D spheroids of MCF-7 breast carcinoma cells by neutrophil-derived cathepsin G and elastase. Cancer Sci., 96, 560570 (2005)

7) Kudo T, Kigoshi H, Hagiwara T, Takino T, Yamazaki M, Yui S. Cathepsin G, a neutrophil protease, induces compact cell-cell adhesion in MCF-7 human breast cancer cells. Mediators Inflamm., 2009, 850940 (2009)

8) Morimoto-Kamata R, Mizoguchi S, Ichisugi T, Yui S. Cathepsin G induces cell aggregation of human breast cancer MCF-7 cells via a 2-step mechanism: catalytic site-independent binding to the cell surface and enzymatic activity-dependent induction of the cell aggregation. Mediators Inflamm., 2012, 456462 (2012).

9) Morimoto-Kamata R, Yui S. Insulin-like growth factor-1 signaling is responsible for cathepsin G-induced aggregation of breast cancer MCF-7 cells. Cancer Sci., 108, 1574-1583 (2017).

10) Pollak M. Insulin and insulin-like growth factor signaling in neoplasia. Nat. Rev. Cancer, 8, 915-928 (2008).

11) Brahmkhatri VP, Prasanna C, Atreya HS. Insulin-like growth factor system in cancer: novel targeted therapies. Biomed. Res. Int., 2015, 538019 (2015).

12) Christopoulos PF, Msaouel P, Koutsilieris M. The role of the insulin-like growth factor-1 system in breast cancer. Mol. Cancer, 14, 43 (2015)

13) Allard JB, Duan C. IGF-binding proteins: why do they exist and why are there so many? Front. Endocrinol. (Lausanne), 9, 117 (2018).

14) Peyrat JP, Bonneterre J, Vennin PH, Jammes H, Beuscart R, Hecquet B, Djiane J, Lefebvre J, Demaille A. Insulin-like growth factor 1 receptors (IGF1-R) and IGF1 in human breast tumors. J. Steroid Biochem. Mol. Biol., 37, 823-827 (1990).

15) Firth SM, Baxter RC. Cellular actions of the insulin-like growth factor binding proteins. Endocr. Rev., 23, 824-854 (2002).

16) Mark S, Kübler B, Höning S, Oesterreicher S, John H, Braulke T, Forssmann WG, Ständker L. Diversity of human insulin-like growth factor (IGF) binding protein-2 fragments in plasma: primary structure, IGF-binding properties, and disulfide bonding pattern. Biochemistry, 44, 3644-3652 (2005).

17) Kibbey MM, Jameson MJ, Eaton EM, Rosenzweig SA. Insulin-like growth factor binding protein-2: contributions of the C-terminal domain to insulin-like growth factor-1 binding. Mol. Pharmacol., 69, $833-845$ (2006).

18) Kuang Z, Yao S, McNeil KA, Thompson JA, Bach LA, Forbes BE, Wallace JC, Norton RS. Cooperativity of the N- and C-terminal domains of insulin-like growth factor (IGF) binding protein 2 in IGF binding. Biochemistry, 46, 13720-13732 (2007).

19) Rajah R, Katz L, Nunn S, Solberg P, Beers T, Cohen P. Insulin-like growth factor binding protein (IGFBP) proteases: functional regulators of cell growth. Prog. Growth Factor Res., 6, 273-284 (1995).

20) Bunn RC, Fowlkes JL. Insulin-like growth factor binding protein proteolysis. Trends Endocrinol. Metab., 14, 176-181 (2003).

21) Gibson TL, Cohen P. Inflammation-related neutrophil proteases, cathepsin $\mathrm{G}$ and elastase, function as insulin-like growth factor binding protein proteases. Growth Horm. IGF Res., 9, 241-253 (1999).

22) Yui S, Osawa Y, Ichisugi T, Morimoto-Kamata R. Neutrophil cathepsin $\mathrm{G}$, but not elastase, induces aggregation of MCF-7 mammary carcinoma cells by a protease activity-dependent cell-oriented mechanism. Mediators Inflamm., 2014, 971409 (2014).

23) McGuire WL Jr, Jackson JG, Figueroa JA, Shimasaki S, Powell $\mathrm{DR}$, Yee D. Regulation of insulin-like growth factor-binding protein (IGFBP) expression by breast cancer cells: use of IGFBP-1 as an inhibitor of insulin-like growth factor action. J. Natl. Cancer Inst., 84, 1336-1341 (1992).

24) Houghton AM, Rzymkiewicz DM, Ji H, Gregory AD, Egea EE, Metz HE, Stolz DB, Land SR, Marconcini LA, Kliment CR, Jenkins KM, Beaulieu KA, Mouded M, Frank SJ, Wong KK, Shapiro SD. Neutrophil elastase-mediated degradation of IRS-1 accelerates lung tumor growth. Nat. Med., 16, 219-223 (2010).

25) Gregory AD, Hale P, Perlmutter DH, Houghton AM. Clathrin pitmediated endocytosis of neutrophil elastase and cathepsin G by cancer cells. J. Biol. Chem., 287, 35341-35350 (2012).

26) Lemmon MA, Schlessinger J. Cell signaling by receptor tyrosine kinases. Cell, 141, 1117-1134 (2010).

27) Kavran JM, McCabe JM, Byrne PO, Connacher MK, Wang Z, Ramek A, Sarabipour S, Shan Y, Shaw DE, Hristova K, Cole PA, Leahy DJ. How IGF-1 activates its receptor. eLife, 3, e03772 (2014).

28) Frattali AL, Treadway JL, Pessin JE. Transmembrane signaling by the human insulin receptor kinase. Relationship between intramolecular $\beta$ subunit trans- and cis-autophosphorylation and substrate kinase activation. J. Biol. Chem., 267, 19521-19528 (1992).

29) Leef JW, Larner J. Insulin-mimetic effect of trypsin on the insulin receptor tyrosine kinase in intact adipocytes. J. Biol. Chem., 262, 14837-14842 (1987).

30) Nakajima K, Powers JC, Ashe BM, Zimmerman M. Mapping the extended substrate binding site of cathepsin $\mathrm{G}$ and human leukocyte elastase. Studies with peptide substrates related to the $\alpha 1$-protease inhibitor reactive site. J. Biol. Chem., 254, 4027-4032 (1979).

31) McRae B, Nakajima K, Travis J, Powers JC. Studies on reactivity of human leukocyte elastase, cathepsin $\mathrm{G}$, and porcine pancreatic elastase toward peptides including sequences related to the reactive site of $\alpha 1$-protease inhibitor ( $\alpha 1$-antitrypsin). Biochemistry, 19, 3973-3978 (1980).

32) Tanaka T, Minematsu Y, Reilly CF, Travis J, Powers JC. Human leukocyte cathepsin G. Subsite mapping with 4-nitroanilides, chemical modification and effect of possible cofactors. Biochemistry, 24, 2040-2047 (1985).

33) Réhault S, Brillard-Bourdet M, Juliano MA, Juliano L, Gauthier F, Moreau T. New, sensitive fluorogenic substrates for human cathepsin $\mathrm{G}$ based on the sequence of serpin-reactive site loops. J. Biol. Chem., 274, 13810-13817 (1999).

34) Hurt B, Schulick R, Edil B, El Kasmi KC, Barnett C Jr. Cancerpromoting mechanisms of tumor-associated neutrophils. Am. J. Surg., 214, 938-944 (2017).

35) Sato T, Takahashi S, Mizumoto T, Harao M, Akizuki M, Takasugi M, Fukutomi T, Yamashita J. Neutrophil elastase and cancer. Surg. Oncol., 15, 217-222 (2006).

36) Maxwell $P$, van den Berg HW. Changes in the secretion of insulinlike growth factor binding proteins- 2 and -4 associated with the development of tamoxifen resistance and estrogen independence in human breast cancer cell lines. Cancer Lett., 139, 121-127 (1999).

37) Akkiprik M, Nicorici D, Cogdell D, Jia YJ, Hategan A, Tabus I, Yli-Harja O, Yu D, Sahin A, Zhang W. Dissection of signaling pathways in fourteen breast cancer cell lines using reverse-phase protein lysate microarray. Technol. Cancer Res. Treat., 5, 543-551 (2006).

38) Probst-Hensch NM, Steiner JH, Schraml P, Varga Z, Zürrer-Härdi U, Storz M, Korol D, Fehr MK, Fink D, Pestalozzi BC, Lütolf UM, Theurillat JP, Moch H. IGFBP2 and IGFBP3 protein expressions in human breast cancer: association with hormonal factors and obesity. Clin. Cancer Res., 16, 1025-1032 (2010).

39) Ruan W, Kleinberg DL. Insulin-like growth factor I is essential for terminal end bud formation and ductal morphogenesis during mammary development. Endocrinology, 140, 5075-5081 (1999). 
40) Pollak MN, Schernhammer ES, Hankinson SE. Insulin-like growth factors and neoplasia. Nat. Rev. Cancer, 4, 505-518 (2004).

41) Pollak M. The insulin and insulin-like growth factor receptor family in neoplasia: an update. Nat. Rev. Cancer, 12, 159-169 (2012).

42) Pickard A, McCance DJ. IGF-binding protein 2-oncogene or tumor suppressor? Front. Endocrinol., 6, 25 (2015).

43) Weroha SJ, Haluska P. The insulin-like growth factor system in cancer. Endocrinol. Metab. Clin. North Am., 41, 335-350 (2012).

44) Busund LT, Richardsen E, Busund R, Ukkonen T, Bjørnsen T, Busch C, Stalsberg H. Significant expression of IGFBP2 in breast cancer compared with benign lesions. J. Clin. Pathol., 58, 361-366 (2005).
45) Wang H, Rosen DG, Wang H, Fuller GN, Zhang W, Liu J. Insulinlike growth factor- binding protein 2 and 5 are differentially regulated in ovarian cancer of different histologic types. Mod. Pathol., 19, 1149-1156 (2006).

46) Fukushima $T$, Kataoka $H$. Roles of insulin-like growth factor binding protein-2 (IGFBP-2) in glioblastoma. Anticancer Res., 27 (6A), 3685-3692 (2007).

47) Azar WJ, Zivkovic S, Werther GA, Russo VC. IGFBP-2 nuclear translocation is mediated by a functional NLS sequence and is essential for its pro-tumorigenic actions in cancer cells. Oncogene, $\mathbf{3 3}$, 578-588 (2014). 